\title{
Use of Fluorescence Spectroscopy to Elucidate RNA Folding Pathways
}

In this unit, some of the practical aspects of performing fluorescence-detected experiments on RNA are described. Fluorescence spectroscopy has proven to be a powerful tool for studying RNA folding, (for examples, see Bevilacqua et al., 1992; Clegg et al., 1992; Turner et al., 1996; Walter and Burke, 1997; Walter et al., 1998, 1999; Silverman and Cech, 1999). Either the ribozyme or a small oligonucleotide substrate may be labeled with a fluorescent probe. Fluorescence spectroscopy affords detection of rapid and slow kinetic processes, with real time acquisition of data.

The first step for performing fluorescencedetected RNA folding experiments involves preparation of modified RNA. A discussion of the advantages and disadvantages of various pendant probes and nucleotide analogs is presented. Then, methods for observing changes in fluorescence are discussed. Some considerations on instrument setup and samples to test are provided. Control experiments that should be performed to assess whether the modification has perturbed the system are also discussed. A variety of kinetic, thermodynamic, and structure-probing experiments are suggested. Lastly, an overview of experiments for obtaining kinetic parameters are presented. General experimental methods and designs for accurately obtaining microscopic rate constants are discussed.

\section{PREPARATION OF \\ FLUORESCENTLY LABELED RNA}

The four natural bases in RNA are not sufficiently fluorescent for most applications; therefore, a fluorophore must be covalently attached to the RNA for study. In the case of two-piece systems involving a large ribozyme and a short substrate, the fluorophore may be engineered into the ribozyme or the substrate. The particular site of attachment is dictated by the scientific question of interest. The chosen fluorophore should have a strong excitation peak that does not overlap with the four bases. In general, excitation anywhere above $310 \mathrm{~nm}$ is preferable. Fluorophores used in RNA folding experiments may be separated into two classifications: pendant probes and nucleotide analogs.

\section{Pendant Probes}

Pendant probes such as fluorescein and pyrene can provide large changes in fluorescence emission upon folding (up to 25 -fold has been observed in the case of pyrene). They may be readily attached to the ends of large or small RNAs prepared enzymatically or by chemical synthesis. For example, the 5' end of RNA may be modified with a thiophosphate by suitable priming of the T7 RNA polymerase transcription of RNA. A wide variety of commercially available thiol-reactive probes may then be coupled to the RNA; Molecular Probes (http://www.probes.com) is an excellent source. Alternatively, oligonucleotides can be synthesized with a $5^{\prime}$ amino group and conjugated to an amine-reactive derivative of a fluorescent dye (Kierzek et al., 1993). The 3' end of the RNA may be modified by reaction with periodate to ring open the cis-diol to a dialdehyde. A variety of amine-containing fluorophores can then be coupled to the $3^{\prime}$ end of the RNA (Reines and Cantor, 1974). Coupling reactions should include a control in which an unmodified RNA is used, and lack of reactivity with the probe should be confirmed. Specific conditions for modifications can be found in the literature from Molecular Probes.

Pendant probes can also be inserted internally in the RNA. A short oligomer can be chemically synthesized with a $2^{\prime}$ amino or thiophosphate (available from Dharmacon; http://www.dharmacon.com) and then coupled to a pendant probe. Modified oligomers can be inserted into a large RNA by ligation. Alternatively, coupling of the probe to the modified RNA could be performed after ligation. Coupling before ligation offers the advantage of easier purification of a modified RNA that is short, although it has the potential disadvantage of interfering with the ligation reaction if it is near the site of ligation.

Several factors can help guide the choice of a suitable fluorophore. Experiments with modified RNA have shown that pyrene emission is strongly quenched by pyrimidines (Kierzek et al., 1993; Manoharan et al., 1995; Netzel et al., 1995), while fluorescein emission is strongly quenched by purines (Walter and Burke, 1997). Thus, if a stretch of pyrimidines changes from single to double stranded upon folding, then attachment of a nearby pyrene may be ideal. In

Contributed by Philip C. Bevilacqua and Douglas H. Turner

RNA Folding 
general, however, there is no way to know for certain if a fluorophore will have favorable properties. A wide variety of reactive probes are commercially available; thus, once an RNA with a reactive group has been prepared, a series of fluorophores should be tested with it.

An important consideration is the length of the linker arm used to attach the fluorophore to the RNA. Short linkers provide the least opportunity for interacting at distant sites, intercalating into a helix, and perturbing the system. Moreover, changes in fluorescence for probes attached by a short linker arm are more likely to reflect changes in local environment, such as protection from solvent. Longer linker arms could be advantageous in cases where the $5^{\prime}$ and $3^{\prime}$ ends are far removed from the site of folding. Potential for intercalation of the probe into a duplex involving the strand to which it is attached can be judged from molecular modeling of the duplex, including molecular dynamics simulations and energy minimization using commercially available computer programs.

Disadvantages of pendant probes include the potential for perturbing the folding process by interacting with neighboring helices in an undesirable fashion, and the inability to directly interpret changes in fluorescence in structural detail.

\section{Nucleotide Analogs}

Nucleotide analogs can be incorporated into oligomers by chemical synthesis. Modified oligomers can then be used directly in experiments, or covalently attached to a large RNA with DNA ligase. Useful analogs include epsilon adenosine $(\varepsilon A)$ and 2 -amino purine. For example, positioning of $\varepsilon A$ on the $5^{\prime}$ or $3^{\prime}$ end of a pyrimidine substrate of a ribozyme led to 2-fold changes in fluorescence upon binding (Sugimoto et al., 1989; Profenno et al., 1997). The analog 2-amino purine has an $\sim 2$-fold decrease in fluorescence upon base pairing (Menger et al., 1996). Nucleotide analogs offer the advantages that they minimize the potential for perturbing the system, and provide localized information about changes in RNA folding (Jean and Hall, 2001).

Several disadvantages exist for nucleotide analogs. In general, the fluorescence sensitivity is lower than for pendant probes, and the change in fluorescence is roughly 10-fold lower (Cantor and Schimmel, 1980). This can lead to poor signal-to-noise ratios and a need for large amounts of sample. Other drawbacks include the extra steps needed for chemical synthesis of modified RNA, although reliable commer- cial sources for synthetic RNA are now available (Dharmacon).

\section{Purification of Labeled \\ Oligoribonucleotides}

Labeled oligoribonucleotides should be purified by HPLC on a $\mathrm{C} 8$ column and stored at $-20^{\circ} \mathrm{C}$ in an EDTA-containing buffer such as TE, pH 7.5 (APPENDIX 2A). Periodically, the RNA should be monitored by HPLC (UNIT 10.5), TLC (APPENDIX $3 D$ ), or polyacrylamide gel electrophoresis (UNIT 10.4) to check for degradation, and the fluorescence spectrum should be checked for any alteration. As when working with any RNA sample, latex gloves should be worn and reagents should be RNase-free. Often, large RNAs are refolded or renatured before use, in case they have been trapped in a non-native conformation during purification and storage. For example, one renaturation involves heating the RNA for $10 \mathrm{~min}$ at $50^{\circ} \mathrm{C}$ in the presence of $\mathrm{Mg}^{2+}$, and another involves heating the RNA for $3 \mathrm{~min}$ at $95^{\circ} \mathrm{C}$ in the absence of $\mathrm{Mg}^{2+}$ followed by slow cooling on the bench. Renaturations should be carried out the day of the experiment, and only the RNA to be used that day should be renatured.

To prevent sticking of hydrophobic probes, microcentrifuge tubes and pipet tips should be silanized before use (see APPENDIX 2A).

\section{MEASURING CHANGES IN FLUORESCENCE}

The first experiment should test whether there is a change in fluorescence upon folding. A fluorometer with emission and excitation monochromators is used, and wavelengths are chosen that give maxima in excitation and emission. Opening the slits to the monochromators can improve the signal-to-noise ratio for the following experiment. A thermostatted sample holder that requires a small volume (several hundred microliters) is ideal. A series of substrates should be tested to look for those that have a large change in fluorescence upon folding. RNA folding or unfolding is initiated and the time dependence of any fluorescence change is measured. Folding or unfolding may be initiated by the addition of substrate or by the addition or removal of divalent ions or a chemical denaturant such as urea. With practice, the dead time for mixing can be as little as 5 to $10 \mathrm{sec}$. Once the system no longer shows a change in fluorescence, an emission spectrum is taken and the change in fluorescence intensity and any wavelength shift are determined. Absence of a change in fluorescence shows that

\subsection{2}


the probe is not good for measuring equilibrium events, but it remains possible that an intermediate state with a different fluorescence emission exists; this can be tested by stopped-flow mixing.

After a probe with a change in fluorescence has been identified, the kinetics of the system should be examined. Processes that occur over nanoseconds to hours can be monitored by fluorescence (Table 11.8.1). Processes with half-lives, $t_{1} / 2$, as fast as $1 \mathrm{nsec}$ can be studied by temperature-jump methods; processes with $t 1 / 2$ from $1 \mathrm{msec}$ to $1 \mathrm{~min}$ can be studied by stopped-flow mixing methods; and processes with $t_{1 / 2}$ as fast as $1 \mathrm{~min}$ can be studied by manual mixing. The temperature-jump method has the advantage that the same sample can be used for many runs in order to increase the signal-to-noise ratio.

\section{Instrumentation: Mixing Methods}

Experiments performed by the authors employed a KinSim stopped-flow mixing apparatus. Fluorescence emission should be collected through either a bandpass or a cut-off filter to maximize the signal that reaches the photomultiplier tube (PMT). Alternatively, stoppedflow apparatuses exist that acquire a rapid emission spectrum. This method has the potential to reveal intermediate states with altered spectra, but has the disadvantage that the signal is weaker and must be averaged over more shots, thus requiring more sample. Sample requirements are modest, however, and a few hundred microliters can allow three or more shots to be averaged. The entire apparatus is thermostatted, allowing the temperature dependence of the kinetics to be determined. Attention should be given to the possibility of photobleaching the sample. A mock sample can be exposed to the light beam to look for any changes in fluorescence. Photobleaching can often be reduced by using a less powerful lamp, by narrowing the monochromator slits, or by closing the slits between time points. If it cannot be fully eliminated, photobleaching should be corrected by subtracting a suitable blank.
Testing if the System is Perturbed

Most researchers are interested in observing the folding properties of unperturbed systems. Thus, attention must be paid to the possibility of perturbing the system of study. An ideal test for perturbation is an activity assay such as bond cleavage by a ribozyme. Modified and unmodified substrate can be radiolabeled and $k_{\text {cat }} / K_{\mathrm{m}}$, the specificity constant, can be determined under various conditions (where $k_{\text {cat }}$ is the observed first-order rate constant under conditions of substrate saturation and $K_{\mathrm{m}}$ is the Michaelis constant). The binding of modified and unmodified substrates to complementary oligomers can be compared to test perturbation of secondary structure formation. Methods of testing include UV melting to look at thermodynamic parameters and stopped-flow mixing to look at kinetic parameters. Folding of the ribozyme can be tested by structure mapping experiments such as chemical modification and enzymatic cleavage. Overall changes in folding may be revealed by circular dichroism (CD). In particular, CD should be measured between 220 and $300 \mathrm{~nm}$ (where the RNA absorbs) and over the wavelength region where the fluorophore absorbs. Ellipticity in the region of fluorophore absorbance implies that the fluorophore is in a chiral environment and may be directly interacting with the RNA.

Large changes in cleavage or binding rate constants, thermodynamic parameters, or RNA structure suggest that the probe is not ideal for the particular system. The choice of probe should be reevaluated. Varying the site of attachment, linker arm, and type of fluorophore should be considered.

\section{Obtaining Kinetic Parameters}

Once a nonperturbing fluorophore that is sensitive to the environment has been prepared, the kinetics of folding may be monitored. A number of articles describing the details of such experiments have been published (see Johnson, 1992). This section presents a brief introduction to the types of experiments that can be

Table 11.8.1 Methods for Studying Folding Kinetics by Fluorescence Detection

\begin{tabular}{lll}
\hline Timescale $\left(t_{1 / 2}\right)$ & Method & Reference \\
\hline $1 \mathrm{nsec}$ & Lifetime & Walter et al. (2001) \\
$\geq 1 \mathrm{nsec}$ & Temperature-jump & Eigen and DeMaeyer (1963); Turner (1986) \\
$1 \mathrm{msec}$ to $1 \mathrm{~min}$ & Stopped-flow mixing & Johnson (1992) \\
$>1 \mathrm{~min}$ & Manual mixing & \\
\hline
\end{tabular}


performed and the information on rate constants that can be obtained.

One of the first experiments to perform is to initiate the RNA folding process and observe the time dependence of fluorescence change. For two-piece systems, such as ribozyme-substrate systems, the kinetics are greatly simplified if pseudo-first-order binding conditions are maintained. This requires having one reagent in large excess. In general, the largest change in signal will be observed if the fluorescent molecule is the limiting reagent. If, however, the fluorophore undergoes a very large change in fluorescence, as in the case of pyrene-labeled oligomers, this condition is not absolutely necessary. Two-piece systems offer the additional advantage that the rate of binding can be adjusted to some extent by varying concentrations. If, for example, the rate is too fast to be observed, concentrations can be lowered to slow the rate of binding. The signal, however, will diminish if ribozyme concentration drops below the equilibrium dissociation constant $\left(K_{\mathrm{d}}\right)$ for the interaction.

Special attention should be paid to experiments in which the ribozyme is maintained in excess over the substrate. Under these conditions, the multimerization state of the large RNA should be monitored by native gel electrophoresis. For example, under certain conditions, the Tetrahymena ribozyme can dimerize, and a large pseudoknot has also been seen to dimerize (P.C. Bevilacqua and D.H. Turner, unpub. observ.; P. Babitzke, P.C. Bevilacqua, and J.E. Schaak, unpub. observ.). Such dimerization processes may lead to further changes in fluorescence. Typically, dimerization can be avoided by renaturing the RNA at higher temperatures prior to the experiment. The effect of various temperatures and salts on the oligomerization state of the RNA can be monitored by native gel electrophoresis of a radiolabeled sample.

Once changes in fluorescence as a function of time are obtained, the data are fit to a firstor higher-order exponential equation to obtain observed rate constants. Because data are generated in real time and acquired on a computer, it is practical to fit the data immediately after mixing. Observed rate constants are then measured at various concentrations of the excess reagent. Plots of observed rate constants versus concentration are fit to curves dictated by a

Use of Fluorescence Spectroscopy to Elucidate RNA Folding Pathways 11.8.4 particular kinetic mechanism to obtain microscopic rate constants. By monitoring the concentration dependence of the observed rate constants, further experiments can be rationally designed and carried out in rapid fashion. The data may also be globally fit by numerical integration to obtain the rate constants using the programs KINSIM and FITSIM (Barshop et al., 1983; Zimmerle and Frieden, 1989; Frieden, 1993). Rate constants obtained by graphical and global fitting methods should be in reasonable agreement. The interested reader is referred to an excellent review containing numerous schemes and their rate equations (Johnson, 1992). Through these approaches, it is possible to accumulate data consistent with a binding mechanism involving numerous uniand bimolecular steps. In many cases, dissociation rate constants are too small to be obtained by such experiments and require use of the pulse-chase experiment.

In the pulse-chase experiment, a fluorescent substrate is in equilibrium with a ribozyme in one mixing syringe (the pulse), and excess amounts of unlabeled substrate are present in the other syringe (the chase). Upon mixing, dissociation of the labeled substrate from the ribozyme is effectively irreversible since the binding site for the labeled molecule is now occupied by an unlabeled molecule. Data are fit to a single exponential equation to obtain a rate constant for dissociation that can be either a microscopic rate constant or a mathematical combination of several rate constants. Data should be obtained at several concentrations of chase to ensure that the observed rate constant is for substrate dissociation and is not dependent on chase concentration. In cases where the $y$ intercept is close to zero for mixing experiments, pulse-chase experiments can allow determination of all the individual rate constants. In cases where the $y$ intercept is well determined by mixing experiments, pulse-chase experiments confirm the value of the dissociation rate constant and allow its direct determination in a single experiment. For example, in the case of pyrene substrate binding to the Tetrahymena ribozyme, the rate constant was the rate of undocking of the P1 duplex-a tertiary RNA unfolding event (Bevilacqua et al., 1992). By varying parameters such as temperature, salt, and $\mathrm{pH}$ during the pulse-chase experiment, insight into the thermodynamic and structural details of the unfolding event may be obtained.

An interesting variation of the pulse-chase experiment is the so-called reversed pulsechase experiment, in which an unlabeled substrate is forced to dissociate from the ribozyme by addition of excess labeled substrate. Because the labeled substrate is in excess, it must have a large change in fluorescence to be ob- 
servable. The rate of dissociation of unlabeled substrate is then directly visualized by the increase in fluorescence upon binding of labeled substrate. Rates may be compared for the pulsechase and reversed pulse-chase experiments to look for any effects of fluorophore upon binding (Bevilacqua et al., 1993). Moreover, such experiments offer the advantage that dissociation behavior of a variety of chemically modified unlabeled substrates can be measured with a single fluorescent substrate.

\section{CONCLUSION}

Fluorescence spectroscopy is a powerful method for studying the folding of large RNAs and offers a number of unique advantages. A variety of RNA folding processes may be monitored by fluorescence detection, including secondary and tertiary structure formation. For example, fluorescence detection has been used to look at binding of oligomer substrate to a ribozyme, and the mechanism was determined to involve base pairing of the substrate followed by docking of the helix into the core of the ribozyme (Bevilacqua et al., 1992). In addition, rates for bond cleavage have been determined by fluorescence detection. Data acquisition is in real time and processes that occur over nanoseconds to hours may be monitored. Fluorescence spectroscopy is a sensitive method; depending on the quantum yield of fluorophore, concentrations down to the nanomolar regime can be used. True equilibrium constants can be measured by fluorescence methods - an advantage over nonequilibrium methods such as gel mobility shift assays. Determination of equilibrium constants is an essential part of determining RNA folding mechanisms, and accurate thermodynamic parameters have allowed insight into the details of RNA tertiary folding.

Limitations of fluorescence spectroscopy include the low level of detail of structural information that may be inferred. Other methods described in this series, such as hydroxyl radical cleavage and chemical modification, provide considerably more insight into detailed structural changes in the RNA.

Perhaps the most difficult and time-consuming part of fluorescence experiments on RNA is identifying a fluorescent probe with favorable properties. An ideal probe should have a large change in fluorescence and minimal perturbation of RNA properties. Once a suitable fluorescent RNA is prepared, however, data may be obtained quickly for rapid and slow kinetic processes. The wide variety and increas- ing number of commercially available fluorophores makes it feasible to rapidly prepare a large series of end-labeled RNAs for examination.

\section{LITERATURE CITED}

Barshop, B.A., Wrenn, R.F., and Frieden, C. 1983. Analysis of numerical methods for computer simulation of kinetic processes: Development of KINSIM: A flexible, portable system. Anal. Biochem. 130:134-145.

Bevilacqua, P.C., Kierzek, R., Johnson, K.A., and Turner, D.H. 1992. Dynamics of ribozyme binding of substrate revealed by fluorescence-detected stopped-flow methods. Science 258:13551358.

Bevilacqua, P.C., Johnson, K.A., and Turner, D.H. 1993. Cooperative and anticooperative binding to a ribozyme. Proc. Natl. Acad. Sci. U.S.A. 90:8357-8361.

Cantor, C.R. and Schimmel, P.R. 1980. Biophysical Chemistry Part II: Techniques for the Study of Biological Structure and Function. pp. 433-465. W.H. Freeman and Company, New York.

Clegg, R.M., Murchie, A.I., Zechel, A., Carlberg, C., Diekmann, S., and Lilley, D.M. 1992. Fluorescence resonance energy transfer analysis of the structure of the four-way DNA junction. Biochemistry 31:4846-4856.

Eigen, M. and DeMaeyer, L. 1963. Relaxation methods. In Techniques of Organic Chemistry, Vol. VIII, Part 2 (S.L. Friess, E.S. Lewis, and A. Weissberger, eds.), pp. 895-1054. John Wiley \& Sons, New York.

Frieden, C. 1993. Numerical integration of rate equations by computer. Trends Biochem. Sci. 18:58-60.

Jean, J.M. and Hall, K.B. 2001. 2-Aminopurine fluorescence quenching and lifetimes: Role of base stacking. Proc. Natl. Acad. Sci. U.S.A. 98:37-41.

Johnson, K.A. 1992. Transient state kinetic analysis of enzyme reaction pathways. In The Enzymes, Vol. 20 (D. Sigman, ed.), pp. 1-61. Academic Press, New York.

Kierzek, R., Li, Y., Turner, D.H., and Bevilacqua, P.C. 1993. 5'-Amino pyrene provides a sensitive, non-perturbing fluorescent probe of RNA secondary and tertiary structure formation. J. Am. Chem. Soc. 115:4985-4992.

Manoharan, M., Tivel, K.L., Zhao, M., Nafisi, K., and Netzel, T.L. 1995. Base-sequence dependence of emission lifetimes for DNA oligomers and duplexes covalently labeled with pyrene. Relative electron-transfer quenching efficiencies of A-nucleoside, G-nucleoside, C-nucleoside, and T-nucleoside toward pyrene. J. Phys. Chem. 99:17461-17472.

Menger, M., Tuschl, T., Eckstein, F., and Pörschke, D. 1996. $\mathrm{Mg}(2+)$-dependent conformational changes in the hammerhead ribozyme. Biochemistry 35:14710-14716.
RNA Folding

Pathways

$\mathbf{1 1 . 8 . 5}$

Supplement 9 
Netzel, T.L., Zhao, M., Nafisi, K., Headrick, J., Sigman, M.S., and Eatson, B.E. 1995. Photophysics of 2'-deoxyuridine (dU) nucleosides covalently substituted with either 1-pyrenyl or 1pyrenoyl-observation of pyrene-to-nucleoside charge-transfer emission in 5-(1-pyrenyl)-dU. $J$. Am. Chem. Soc. 117:9119-9128.

Profenno, L.A., Kierzek, R., Testa, S.M., and Turner, D.H. 1997. Guanosine binds to the Tetrahymena ribozyme in more than one step, and its $2^{\prime}-\mathrm{OH}$ and the nonbridging pro-Sp phosphoryl oxygen at the cleavage site are required for productive docking. Biochemistry 36:12477-12485.

Reines, S.A. and Cantor, C.R. 1974. New fluorescent hydrazide reagents for the oxidized 3 '-terminus of RNA. Nucl. Acids Res. 1:767-786.

Silverman, S.K. and Cech, T.R. 1999. RNA tertiary folding monitored by fluorescence of covalently attached pyrene. Biochemistry 38:14224-14237.

Sugimoto, N., Sasaki, M., Kierzek, R., and Turner, D.H. 1989. Binding of a fluorescent oligonucleotide to a circularized intervening sequence for Tetrahymena thermophila. Chem. Lett. (1989):2223-2226.

Turner, D.H. 1986. Temperature-jump methods. In Investigations of Rates and Mechanisms of Reactions, Vol. 6, Part 2 (C. F. Bernasconi, ed.), pp. 141-189. John Wiley \& Sons, New York.

Turner, D.H., Li, Y., Fountain, M., Profenno, L., and Bevilacqua, P.C. 1996. Dynamics of a group I ribozyme detected by spectroscopic methods. In Nucleic Acids and Molecular Biology (F. Eckstein and D.M.J. Lilley, eds.), pp. 19-32. Springer-Verlag, Berlin.

Walter, N.G. and Burke, J.M. 1997. Real-time monitoring of hairpin ribozyme kinetics through basespecific quenching of fluorescein-labeled substrates. RNA 3:392-404.

Walter, N.G., Hampel, K.J., Brown, K.M., and Burke, J.M. 1998. Tertiary structure formation in the hairpin ribozyme monitored by fluorescence resonance energy transfer. EMBO J. 17:23782391.
Walter, N.G., Burke, J.M., and Millar, D.P. 1999. Stability of hairpin ribozyme tertiary structure is governed by the interdomain junction. Nat. Struct. Biol. 6:544-549.

Walter, N.G., Chan, P.A., Hampel, K.J., Millar, D.P., and Burke, J.M. 2001. A base change in the catalytic core of the hairpin ribozyme perturbs function but not domain docking. Biochemistry 40:2580-2587.

Zimmerle, C.T. and Frieden, C. 1989. Analysis of progress curves by simulations generated by numerical integration. Biochem. J. 258:381-387.

\section{INTERNET RESOURCES}

http://chemgenes.com

ChemGenes site with catalog of precursors for synthesis of nucleic acids with fluorescent bases.

http://www.dharmacon.com

Dharmacon Research site with catalog offering synthesis of labeled nucleic acids.

http://www.glenres.com

Glen Research site with catalog of precursors for synthesis of labeled nucleic acids.

http://www.probes.com

Molecular Probes site with catalog and technical information for fluorophores.

http://www.trilinkbiotech.com

TriLink BioTechnologies site with catalog offluorescent nucleoside triphosphates.

Contributed by Philip C. Bevilacqua

The Pennsylvania State University

University Park, Pennsylvania

Douglas H. Turner

University of Rochester

Rochester, New York
Use of

Fluorescence Spectroscopy to Elucidate RNA Folding Pathways

11.8.6 